\title{
Planck view of the M 82 galaxy
}

\author{
V. G. Gurzadyan ${ }^{1,2}$, F. De Paolis ${ }^{3,4}$, A. A. Nucita ${ }^{3,4}$, G. Ingrosso ${ }^{3,4}$, A. L. Kashin ${ }^{2}$, H. G. Khachatryan ${ }^{2}$, S. Sargsyan², \\ G. Yegorian ${ }^{2}$, Ph. Jetzer ${ }^{5}$, A. Qadir ${ }^{6}$, and D. Vetrugno ${ }^{7,8}$
}

\author{
1 SIA, Sapienza University of Rome, 00185 Rome, Italy \\ 2 Center for Cosmology and Astrophysics, Alikhanian National Laboratory and and Yerevan State University, Yerevan, Armenia \\ 3 Dipartimento di Matematica e Fisica "E. De Giorgi", Università del Salento, via per Arnesano, 73100 Lecce, Italy \\ ${ }^{4}$ INFN, Sez. di Lecce, via per Arnesano, 73100 Lecce, Italy \\ e-mail: DePaolis@le.infn.it \\ 5 Physik-Institut, Universität Zürich, Winterthurerstrasse 190, 8057 Zürich, Switzerland \\ 6 School of Natural Sciences, National University of Sciences and Technology, H-12 Islamabad, Pakistan \\ 7 Department of Physics, University of Trento, 38123 Povo, Trento, Italy \\ 8 TIFPA/INFN, 38123 Povo, Italy
}

Received 6 August 2015 / Accepted 24 August 2015

\section{ABSTRACT}

\begin{abstract}
Planck data towards the galaxy M 82 are analyzed in the 70, 100 and $143 \mathrm{GHz}$ bands. A substantial north-south and east-west temperature asymmetry is found, extending up to $1^{\circ}$ from the galactic center. Being almost frequency-independent, these temperature asymmetries are indicative of a Doppler-induced effect regarding the line-of-sight dynamics on the halo scale, the ejections from the galactic center and, possibly, even the tidal interaction with M 81 galaxy. The temperature asymmetry thus acts as a model-independent tool to reveal the bulk dynamics in nearby edge-on spiral galaxies, like the Sunyaev-Zeldovich effect for clusters of galaxies.
\end{abstract}

Key words. galaxies: general - galaxies: individual: M 82 - galaxies: halos

\section{Introduction}

M 82 (NGC 3034, also known as the Cigar galaxy) is the largest galaxy of the M81 group in the Ursa Major constellation. It is nearly edge-on (its inclination angle to the line of sight is $i \simeq 80^{\circ}$ ), and it is the closest galaxy that hosts a starburst nucleus, being at a distance of only 3.5 Mpc (Dalcanton et al. 2009) ${ }^{1}$. Originally classified as a dwarf irregular type-II galaxy (Sandage \& Brucato 1979), M 82 has been more recently reclassified as a late type SBc galaxy after the discovery of a bar (oriented almost along the major axis of the galaxy and with about 1 ' length) and arm structure in its disk (Telesco et al. 1991; Achtermann \& Lacy 1995; Mayya et al. 2005). The coordinates of the M 82 center are RA $(\mathrm{J} 2000)=09^{\mathrm{h}} 55^{\mathrm{m}} 52.7^{\mathrm{s}}, \operatorname{Dec}(\mathrm{J} 2000)=69^{\circ} 40^{\prime} 46^{\prime \prime}$ (corresponding to galactic coordinates $l=141.409498^{\circ}$ and $\left.b=40.567579^{\circ}\right)$. It is one of the most studied galaxies after the Milky Way (see, e.g., Markarian 1962) and has been extensively observed at all wavelengths, from the radio band to high energies (see, e.g., Hutton et al. 2014). In the IR, M 82 is the brightest galaxy in the sky, and shows an outwardly expanding gas (Hutton et al. 2014), which is probably being driven out by the combined emerging particle winds of many stars, together creating a galactic superwind (see also Gandhi et al. 2011). Stellar dynamics measurements of the innermost bulge region of the central M 82 galaxy show that it harbors a supermassive black hole with a mass $\simeq 3 \times 10^{7} M_{\odot}$ (Gaffney et al. 1993).

The M 82 rotation curve has been derived in several studies using both stellar and gaseous tracers up to a radius of about $170^{\prime \prime}(\simeq 2.9 \mathrm{kpc})$. Mayall (1960) and Goetz et al. (2014) obtained a rotation curve using optical emission and absorption lines with

\footnotetext{
1 At the M 82 distance, $1^{\prime}$ corresponds to about $1 \mathrm{kpc}$.
}

a peak value of about $130 \mathrm{~km} \mathrm{~s}^{-1}$, whereas Sofue (1998) used the CO and $\mathrm{H}$ I lines to find a peak value of about $190 \mathrm{~km} \mathrm{~s}^{-1}$. Both analyses find a steeply rising curve, peaking at about $25^{\prime \prime}$ from the galaxy center and rapidly declining in a Keplerian fashion beyond $25^{\prime \prime}$, likely indicating that M 82's extended disk mass is missing, which is very peculiar and exceptional for a disk galaxy. More recently, Greco et al. (2012) have presented a $K$-band spectroscopic study and measured the M 82 rotation curve out to about $4^{\prime}(\simeq 4 \mathrm{kpc})$ on both the eastern and western sides of the galaxy. At variance with the previous studies, it has been found that the stellar rotation velocity is flat on scales of $1-4 \mathrm{kpc}$ (with only a slight decline on the eastern side) with a value of $\simeq 110-120 \mathrm{~km} \mathrm{~s}^{-1}$ out to $4 \mathrm{kpc}$, thus implying an M 82 total dynamical mass of $\sim 10^{10} M_{\odot}$.

As shown by De Paolis et al. (2011, 2014, 2015), cosmic microwave background (CMB) data offer a unique opportunity to study the large-scale temperature asymmetries far beyond the size typically accessible with other tools toward nearby astronomical systems. We study the M 82 galaxy by using Planck data within a galactocentric radius of $1^{\circ}$.

\section{Planck data analysis and results}

The sensitivity, angular resolution, and frequency coverage of its detectors make Planck a powerful instrument for both cosmology and galactic and extragalactic astrophysics (Planck Collaboration I 2015). Here we use the data of Planck 2015 release (Planck Collaboration I 2015) in the bands at $70 \mathrm{GHz}$ of the Low Frequency Instrument (LFI), and in the bands at 100 and $143 \mathrm{GHz}$ of the High Frequency Instrument (HFI). For a review of Planck results and instrument characteristics, we refer the interested reader to Burigana et al. (2013), among others. 


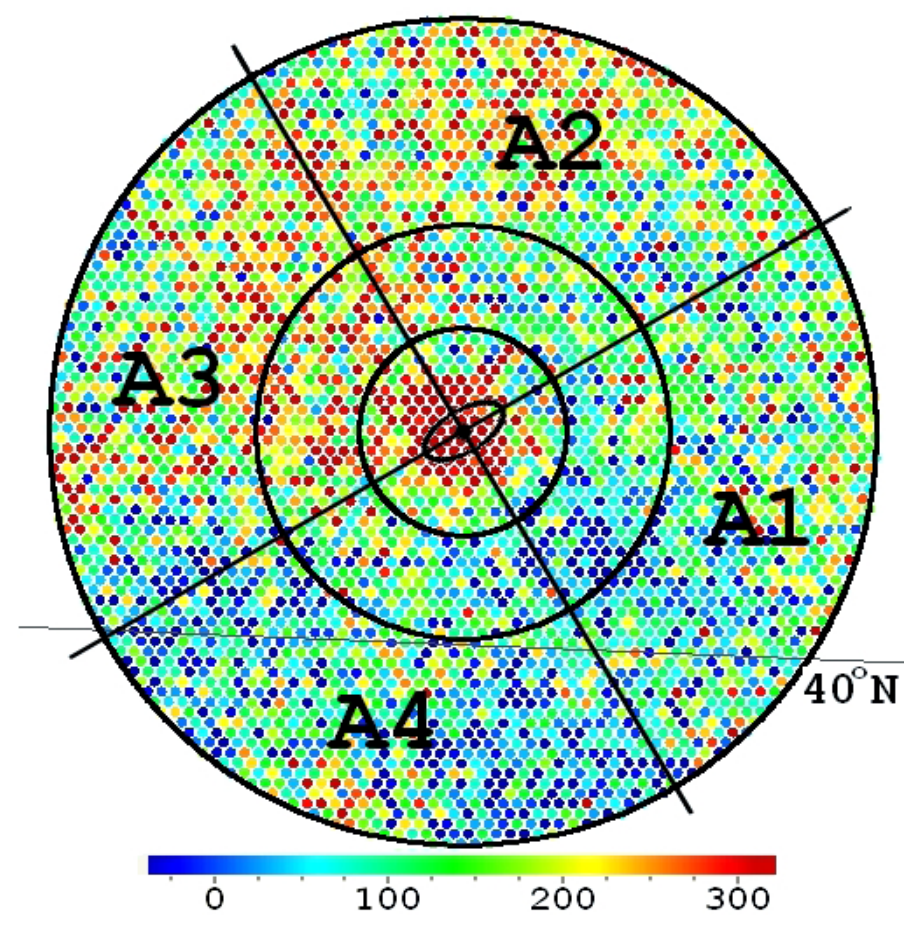

Fig. 1. The Planck field toward the M 82 galaxy in the $70 \mathrm{GHz}$ band. The pixel color gives the temperature excess with respect to the mean CMB temperature in $\mu \mathrm{K}$. The galactocentric radii of the circles are $15^{\prime}$, $30^{\prime}$ and $1^{\circ}$, respectively. The M 82 galaxy is indicated by the inner ellipse with major and minor axes of $10.73^{\prime}$ and $5.02^{\prime}$, respectively. The analysis presented in Sect. 2 is performed within the quadrants indicated as A1, A2, A3, and A4. The line corresponding to Galactic latitude $40^{\circ}$ north is also shown.

Planck's resolution is $13^{\prime}, 9.6^{\prime}$, and $7.1^{\prime}$ in terms of FHWM at 70, 100, and $143 \mathrm{GHz}$ bands, respectively, and frequency maps (Planck Collaboration XVI 2015) are provided in CMB temperature at resolution corresponding to $N_{\text {side }}=2048$ in HEALPix scheme (Górski et al. 2005).

To study the CMB data toward the M 82 galaxy, the considered region of the sky (shown in Fig. 1 in the Planck band at $70 \mathrm{GHz}$ ) has been divided into four quadrants indicated as A1, A2, A3, and A4. The inner ellipse indicates the M 82 galaxy extension in the optical band, while the circles are at galactocentric radii $15^{\prime}, 30^{\prime}$, and $1^{\circ}$, respectively.

The mean temperature excess (with respect to the mean CMB temperature) in $\mu \mathrm{K}$ in each of the indicated regions was obtained in each Planck band at 70, 100, and $143 \mathrm{GHz}$ with the corresponding standard error ${ }^{2}$. As one can see from the upper panel of Fig. 2, the A3+A4 region is systematically hotter than the $\mathrm{A} 1+\mathrm{A} 2$ region with an excess temperature almost constant within $15^{\prime}$ and $30^{\prime}$ in the three Planck bands at 70,100 , and $143 \mathrm{GHz}$. The detected excess with respect to the minor axis of the M 82 galaxy is therefore aligned along the rotational direction, indicating a Doppler induced effect modulated by the spin of the galaxy. However, the temperature asymmetry completely disappears if one considers the data within $1^{\circ}$, and this indicates

\footnotetext{
2 The standard error has been calculated as the standard deviation of the excess temperature distribution divided by the square root of the pixel number in each region. We have verified that within the errors, the sigma values calculated in that way are consistent with those evaluated by using the covariance matrix obtained by a best-fitting procedure with a Gaussian to the same distribution.
}
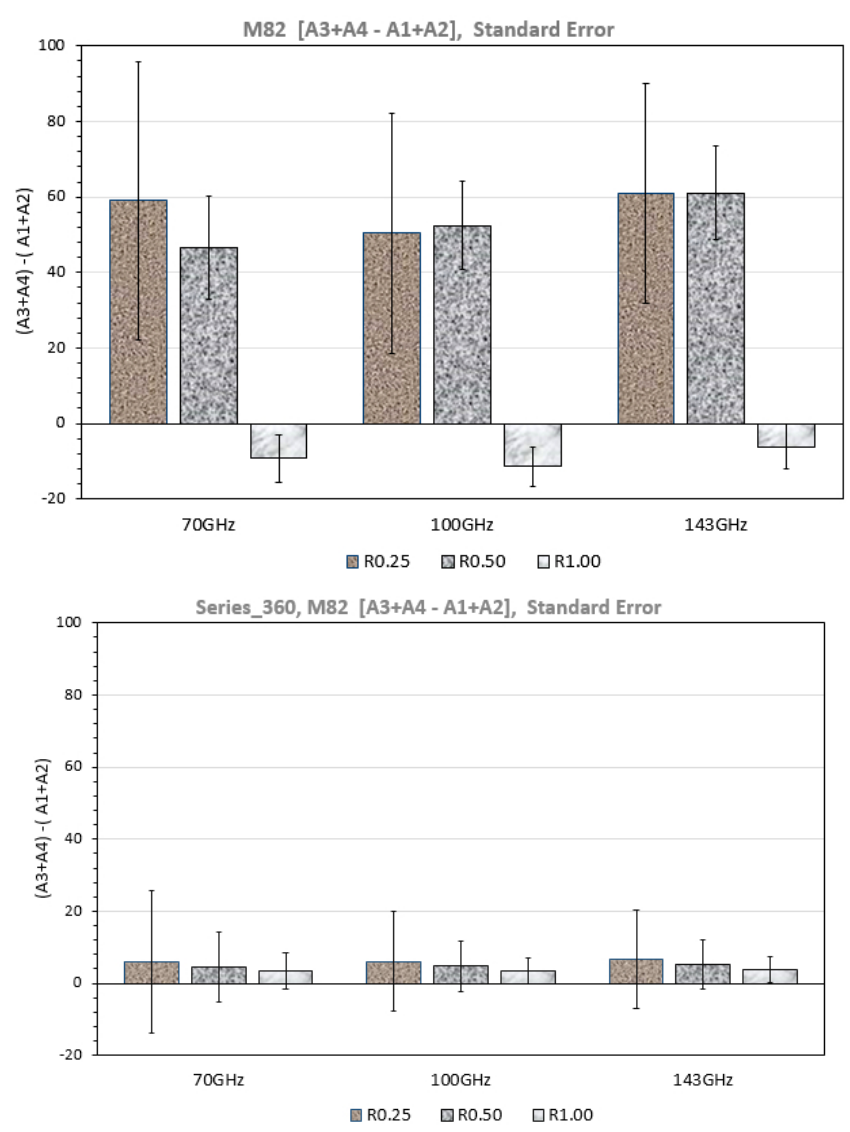

Fig. 2. Upper panel: the excess temperature toward $\mathrm{M} 82$ in $\mu \mathrm{K}$ (with the standard errors) of the $\mathrm{A} 3+\mathrm{A} 4$ region with respect to the $\mathrm{A} 1+\mathrm{A} 2$ region in the three considered Planck bands up to galactocentric radii of $15^{\prime}$ (R0.25), 30' (R0.50), and $1^{\circ}$ (R1.00). Bottom panel: the same for the 360 control fields with the same geometry (shown in Fig. 1) equally spaced at one degree distance to each other in Galactic longitude and at the same latitude as M 82.

that the temperature asymmetry between the two M 82 sides is substantial only up to about $30-40 \mathrm{kpc}$.

To test whether the temperature asymmetry we see is real or can be explained by a random fluctuation of the CMB signal (which is very patchy, especially on angular scales of $0.5^{\circ}-1^{\circ}$ ), we considered 360 control field regions with the same shape as shown in Fig. 1 at the same Galactic latitude of M 82, but at $1^{\circ}$ longitude from each other. For each region, we determined the excess temperature profile and calculated the average profile and corresponding standard deviation. The results are shown in the histogram in the bottom panel of Fig. 2. As one can see, the temperature asymmetry in the 360 control fields in the three considered Planck bands is null (within the standard errors), when integrating within $15^{\prime}, 30^{\prime}$, and $1^{\circ}$. Here we note that the standard errors within $15^{\prime}$ toward the M 82 galaxy are quite large, as expected, owing to the small number of pixels. Therefore, in the three considered bands and within R0.25 (15'), the temperature asymmetry excess is detected within a rather low confidence level (slightly more than 1 standard deviation). The situation becomes better when considering the intermediate circle at $30^{\prime}$ (R0.50). For example, in this case in the $100 \mathrm{GHz}$ band, we detect a temperature excess of $50 \pm 10 \mu \mathrm{K}$, while the control fields give a temperature excess $\leq 12 \mu \mathrm{K}$. Therefore, in this region the temperature asymmetry is detected with a confidence level of about $3 \sigma$.

Next, we consider the temperature asymmetry with respect to the major axis of the M 82 galaxy. In the upper panel of Fig. 3 

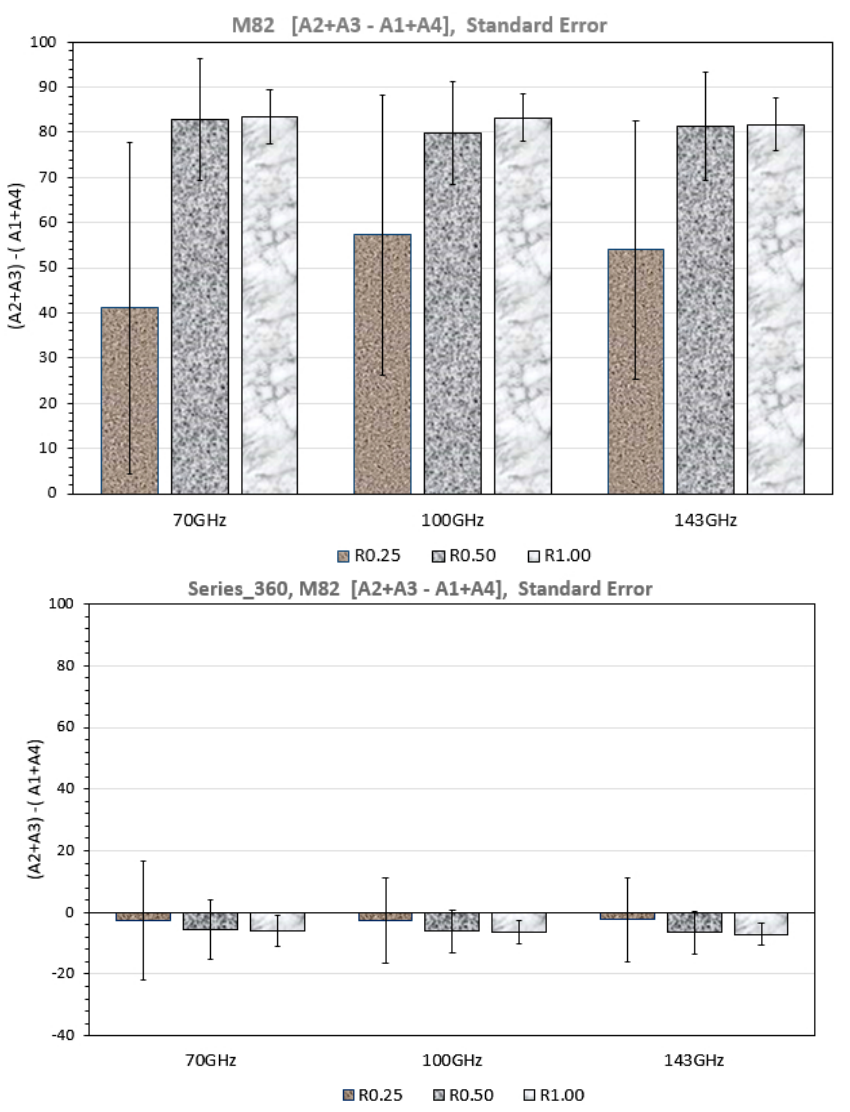

Fig. 3. Upper panel: the excess temperature in $\mu \mathrm{K}$ (with the standard errors) of the $\mathrm{A} 2+\mathrm{A} 3$ region with respect to the $\mathrm{A} 1+\mathrm{A} 4$ region in the three considered Planck bands up to galactocentric radii of $15^{\prime}, 30^{\prime}$, and $1^{\circ}$ toward the M 82 galaxy. Bottom panel: the same for the 360 control fields as in Fig. 2.

we give the $\mathrm{CMB}$ temperature asymmetry of the $\mathrm{A} 2+\mathrm{A} 3$ regions with respect to the other two regions. As in Fig. 2 we also give the temperature asymmetry in the 360 control fields, which is consistent with zero in the three considered bands and within $15^{\prime}$ (R0.25), 30' (R0.50), and $1^{\circ}$ (R1.00). As one can see, the detected temperature asymmetry is substantial (since $\simeq 40 \mu \mathrm{K}$ to $\simeq 80 \mu \mathrm{K})$ in all the Planck bands within at least $1^{\circ}(\simeq 60 \mathrm{kpc})$ of the M 82 center. The very fact that the detected temperature asymmetry is almost frequency independent is a clear and strong indication of an effect of the galaxy rotation.

Before closing this section, we stress here that the procedure we followed to test the reliability of the detected temperature asymmetry, that is, of considering the 360 control regions, is more reliable than simulating the CMB sky maps in each band being considered. While the latter methodology of generating sky maps to estimate the error bars is mandatory when dealing with the whole sky (as in cosmological studies), in our case we are considering only fairly small regions of the Planck sky maps, and the adopted procedure is more reliable since it avoids the ambiguities possibly involved in the simulations.

\section{Conclusions and further perspectives}

We have considered Planck 2015 release data in the bands at 70, 100 , and $143 \mathrm{GHz}$ and detected both a east-west and a northsouth temperature asymmetry toward the M 82 galaxy. We detect a temperature asymmetry of the $\mathrm{A} 3+\mathrm{A} 4$ versus the $\mathrm{A} 1+\mathrm{A} 2$ regions (that is aligned with respect to the M 82 rotation axis).
This is present in all the considered Planck bands up to a galactocentric distance of $30 \mathrm{kpc}$. Even more robust is the temperature asymmetry of the $\mathrm{A} 2+\mathrm{A} 3$ versus the $\mathrm{A} 1+\mathrm{A} 4$ regions, which extends up to at least $60 \mathrm{kpc}$, since it is oriented as the M 82 outflow.

The very fact that the detected temperature asymmetry is almost frequency independent and is approximately in the galaxy spin direction (although probably tilted toward the M 82 outflow direction) is a rather clear indication of a Doppler-induced effect modulated by the spin of the M 82 galaxy. Since the temperature asymmetry is detected far beyond the optical extension of the galaxy, it has to bring the imprint of the M 82 halo, whose rotation axis is probably tilted in the west direction with respect to the spin axis of the disk. This conclusion is reinforced by the observation that the rotation axis of the ionized emission-line gas is offset from the stellar rotation axis and the photometric major axis by an angle of about $12^{\circ}$ (Westmoquette et al. 2009). This has been interpreted as possibly resulting from the interaction of M 82 with the M 81 galaxy (which is placed approximately in the A3 region in Fig. 1 at about 40' distance from the M 82 center) and/or with NGC 3077 (which lies in the A2 region but beyond the $\mathrm{R} 1.00$ circle) in the past.

We also mention that the rotation of the M 82 galaxy up to galactocentric distances of a few kpc has also been studied recently through the kinematics of planetary nebulae (Johnson et al. 2009). The individual radial velocities of these planetary nebulae not only agree with the rotation curve measured with other methods (see the discussion in the Introduction), but moreover, the nebulae at high Galactic latitude (away from the M 82 disk) show a clear rotation signature, in agreement with our results.

Before closing, we would like to note that the Planck Early Release Compton Source Catalogue has been used, together with Wilkinson Microwave Anisotropy Probe (WMAP) data by Peel et al. (2011), to derive the continuum spectrum of the M 82 galaxy. Then, by a least-squares fit to the obtained spectrum by a combination of synchrotron, free-free and thermal dust models, the authors have found evidence of AME (anomalous microwave emission) from this galaxy. However, in a different way from the analysis presented here, the study by Peel et al. (2011) is limited to the optical extension of M 82 (essentially the 1-2 Planck pixels of the ellipse at the center of Fig. 1 ), while our study is a "spatial" analysis of the region around M 82, and, hopefully, brings information from its halo, whose presence has hitherto been expected. Actually, regardless of the emission mechanism that may contribute to the signal at CMB wavelengths ${ }^{3}$, the Doppler-induced temperature asymmetry is given by

$\frac{\Delta T}{T}=2 \tau_{\mathrm{eff}} \frac{v}{c} \sin i$

where $v$ is the galaxy rotation velocity, $i$ the inclination angle of the galaxy rotation axis with respect to the line of sight ${ }^{4}$, and $\tau_{\text {eff }}$ the effective optical depth proper of the particular emission mechanism responsible for the temperature asymmetry. For example, for the $\mathrm{kSZ}$ case $\tau_{\text {eff }}$ is the projected optical depth due to

3 These mechanisms are (i) free-free emission; (ii) synchrotron emission; (iii) anomalous microwave emission (AME) from dust grains; (iv) the kinetic Sunyaev-Zel'dovich (kSZ) effect; and (v) cold gas clouds populating the outer galaxy regions (as first proposed, in the context of the M31 halo, by De Paolis et al. 1995).

4 In the case of the galactic disks, it can be roughly estimated as $i=$ $\arccos (b / a)$, where $b$ and $a$ are the minor and major axes of the galaxy. 
Thomson scattering of the CMB photons on free electrons (see, e.g., Mak et al. 2011), while $\tau_{\text {eff }}=\bar{\tau} S$ in the case of the cold clouds mechanism (where $\bar{\tau}$ is the frequency averaged cloud optical depth and $S$ is the cloud filling factor, as in De Paolis et al. 1995). As a matter of fact and keeping in mind the object of the present study, mechanisms (i), (ii), and (iv), as detailed in footnote 3, require the presence of hot plasma in the M 82 halo which is indeed observed up to about $6 \mathrm{kpc}$ (Bregman et al. 1995) along the minor axis but might also be more extended as shown by Suzaku observations (Tsuru et al. 2007) - while mechanisms (iii) and (v) only involve an extended population of cold gas and/or dust, which is observed as well ${ }^{5}$.

The importance of the methodology we propose, which is applied to the M 82 case, is that in spite of its simplicity, it may allow us to consistently estimate the dynamical mass $M_{\text {dyn }}$ (contained within a certain galactocentric distance $R$ ) of the considered galaxy once the temperature asymmetry has been quantified. In fact,

$$
M_{\mathrm{dyn}}(<R)=\frac{v^{2}}{G} R=700\left(\frac{\Delta T_{\mu K}}{\tau_{\mathrm{eff}} \sin i}\right)^{2} R_{\mathrm{kpc}} M_{\odot}
$$

which directly envisages a lower limit to $M_{\text {dyn }}$ (obtained for $\left.\tau_{\text {eff }}=1\right)$ that, in the case of the M 82 galaxy, turns out to be $(\Delta T \simeq 80 \mu \mathrm{K}$ and $R \simeq 60 \mathrm{kpc}) M_{\text {dyn }} \geq 2.8 \times 10^{8} M_{\odot}$. This is consistent with the observations and can be further improved by considering the details regarding the emission mechanisms involved (that is estimating $\tau_{\text {eff }}$ ). The contribution to the revealed temperature asymmetries can be on different scales from the matter outflow from the galactic center, the halo rotation, and the stripped matter due to M 81's interaction. In general, our method applied to nearby edge-on spirals reveals the galactic halo bulk dynamics on a rather large scale in a model-independent way. In this sense it resembles the SZ effect, which on galactic scales cannot work since the $e^{-}$temperature is not high enough to produce a substantial effect. The importance of the halo's traced parameters (scale, rotation) by this method is also obvious for dark matter and cosmological reasons, especially if complemented by other dynamical and bulk motion information (Rauzy \& Gurzadyan 1998). Used in synergy with other data, this may provide unique keys to studying the bulk dynamics, the outflow ejection processes, and thus the evolution of galactic systems.

Acknowledgements. We acknowledge the use of Planck data in the Legacy Archive for Microwave Background Data Analysis (LAMBDA) and HEALPix (Górski et al. 2005) package. F.D.P., A.A.N., and G.I. acknowledge the support by the INFN project TAsP, and P.J. acknowledges support from the Swiss National Science Foundation. One of us (AQ) is grateful for hospitality to the DST Centre of Excellence in Mathematical \& Statistical Sciences of the University of the Witwatersrand, Johannesburg, South Africa.

\section{References}

Achtermann, J. M., \& Lacy, J. H. 1995, ApJ, 439, 163

Beirão, P., Armus, L., Lehnert, M. D., et al. 2015, MNRAS, 451, 2640

Bregman, J. N., Schulman, E., \& Tomisaka, K. 1995, ApJ, 439, 155

Burigana, C., Davies, R. D., De Bernardis, P., et al. 2013, IJMPD, 22, 1330011

Dalcanton, J. J., Williams, B. F., Seth, A. C., et al. 2009, ApJS, 183, 67

De Paolis, F., Ingrosso, G., Jetzer, Ph., et al. 1995, A\&A, 299, 647

De Paolis, F., Gurzadyan, V. G., Ingrosso, G., et al. 2011, A\&A, 534, L8

De Paolis, F., Gurzadyan, V. G., Nucita, A. A., et al. 2014, A\&A, 565, L3

De Paolis, F., Gurzadyan, V. G., Nucita, A. A., et al. 2015, A\&A, 580, L8

Gaffney, N. I., Lester, D. F., \& Telesco, C. M. 1993, ApJ, 407, L57

Gandhi, P., Isobe, N., Birkinshaw, M., et al. 2011, PASJ, 63, S505

Goetz, M., McKeith, C. D., Downes, D., \& Greve, A., 1990, A\&A, 240, 52

Górski, K. M., Hivon, E., Banday, A. J., et al. 2005, ApJ, 622, 759

Greco, J. P., Martini, P., \& Thompson, T. A. 2012, ApJ, 757, 24

Hutton, S., Ferreras, I., Wu, K., et al., 2014, MNRAS, 440, 150

Johnson, L. C., Méndez, R. H., \& Teodorescu, A. M. 2009, ApJ, 697, 1138

Mak, D. S. M., Pierpaoli, E., \& Osborne, S. J. 2011, ApJ, 736, 116

Markarian, B. E. 1962, Astron. Zh., 39, 1041

Mayya, Y. D., Carrasco, L., \& Luna, A. 2005, ApJ, 628, L33

Mayall, N. U. 1960, Annales d'Astrophysique, 23, 344

Peel, M. W., Dickinson, C., Davies, R. D., et al. 2011, MNRAS, 416, L99

Planck Collaboration I. 2015, A\&A, submitted [arXiv: 1502.01582]

Planck Collaboration XVI. 2015, A\&A, submitted [arXiv: 1506.07135]

Rauzy, S., \& Gurzadyan, V. G. 1998, MNRAS, 298, 114

Sandage, A., \& Brucato, R. 1979, AJ, 84, 472

Sofue, Y. 1998, PASJ, 50, 227

Telesco, C. M., Joy, M., Dietz, K., et al. 1991, ApJ, 369, 135

Tsuru, T. G., Ozawa, M., Hyodo, Y., et al. 2007, PASJ, 59, 269

Veilleux, S., Rupke, D. S. N., \& Swaters, R. 2009, ApJ, 700, 149

Westmoquette, M. S., Smith, L. J., Gallagher, J. S., et al. 2009, ApJ, 696, 192

\footnotetext{
5 We note that $H_{2}$ knots and filaments in the M 82 halo have been detected even beyond $5 \mathrm{kpc}$ (Veilleux et al. 2009; Beirão et al. 2015).
} 